\title{
Optimal 1,3-propanediol production: Exploring the trade-off between process yield and feeding rate variation
}

\author{
Lei Wang ${ }^{\mathrm{a}}$, Qun Lin ${ }^{\mathrm{b}}$, Ryan Loxton ${ }^{\mathrm{b}, \mathrm{c}}$, Kok Lay Teo ${ }^{\mathrm{b}}$, Guanming Cheng ${ }^{\mathrm{a}}$ \\ ${ }^{a}$ School of Mathematical Science, Dalian University of Technology, Dalian, People's Republic of China \\ ${ }^{b}$ Department of Mathematics and Statistics, Curtin University, Perth, Australia \\ ${ }^{c}$ Institute of Cyber-Systems and Control, Zhejiang University, Hangzhou, People's Republic of China
}

\begin{abstract}
This paper proposes a new optimal control model for the production of 1,3-propanediol (1,3-PD) via microbial fedbatch fermentation. The proposed model is governed by a nonlinear multistage dynamic system with two modes: feeding mode, in which glycerol and alkali substrates are added continuously to the fermentor; and batch mode, in which no substrates are added to the fermentor. The non-standard objective function incorporates both the final 1,3PD yield and the cost of changing the input feeding rate, which is the control variable for the fed-batch fermentation process. Continuous state inequality constraints are imposed to ensure that the concentrations of biomass, glycerol, and reaction products lie within specified limits. Using the constraint transcription method, we approximate the continuous state inequality constraints by a conventional inequality constraint to yield an approximate parameter optimization problem. We then develop a combined particle swarm and gradient-based optimization algorithm to solve this approximate problem. The paper concludes with simulation results.
\end{abstract}

Keywords: Nonlinear systems, Multistage dynamic systems, Optimal control, Fed-batch fermentation, Total variation

\section{Introduction}

1,3-Propanediol (1,3-PD) is an organic compound with a wide range of applications in cosmetics, adhesives, lubricants and medicines [1]. Due to its unique symmetrical structure, 1,3-PD can act as a monomer for the production of various industrial polymers, including polyesters and polyurethanes [2]. Production methods for 1,3-PD can be divided into two categories: chemical synthesis and microbial conversion. This paper focuses on the latter category, which is now becoming increasingly attractive in industry because of the cheap availability of renewable feedstock such as glycerol, a byproduct of biodiesel production $[2,3]$.

Glycerol is converted to 1,3-PD via bacterial fermentation $[4,5]$. The fermentation process can be one of three types: batch fermentation (all substrate is present at the beginning of the reaction and nothing is added or removed from the fermentor during the reaction); fed-batch fermentation (fresh medium is added during the reaction to prevent nutrient deple- tion, but nothing is removed); and continuous fermentation (fresh medium is added during the reaction while old medium is removed). This paper focuses on fedbatch fermentation, which is typically implemented by switching between a batch mode (in which the input feed is closed) and feeding mode (in which the input feed is open). Switching between batch and feeding modes in this manner makes it easier to regulate the $\mathrm{pH}$ value for optimal reaction conditions $[6,7,8]$. In addition, substrate inhibition (whereby secondary reaction products hinder the consumption of substrate) is greatly reduced, allowing for more glycerol and alkali to be consumed and thus more biomass to be produced with higher 1,3-PD concentration [9].

The fed-batch fermentation process for converting glycerol to 1,3-PD begins with batch operation $[10,11]$. During this initial batch phase, the biomass tends to grow exponentially. Once the exponential growth phase ends, the glycerol and alkali substrates are added continuously to the reactor to regulate the $\mathrm{pH}$ level. The

Email address: r.loxton@curtin.edu.au (Ryan Loxton) 
process then reverts to batch mode, and so on until the end of the final batch phase.

To achieve commercially-viable concentrations of 1,3-PD, optimization of the microbial conversion process is critical. A major challenge is the presence of undesirable secondary products (acetate and ethanol), which inhibit the production of biomass. To address this challenge, precise mathematical models are required for process control and optimization. Recently in [12], a nonlinear impulsive model was proposed to describe the fed-batch fermentation process for converting glycerol to 1,3-PD. The corresponding parameter identification and optimal control problems were investigated in $[13,14,15,16,17]$. The impulsive model in [12] is based on the assumption that the addition of glycerol and alkali substrates is a discrete process. However, in practice, glycerol and alkali are added continuously, not at discrete times. Thus, a new model involving a nonlinear multistage dynamic system with continuous input variables was proposed in $[18,19,20]$ for describing the fed-batch fermentation process. This model was further investigated in [21].

The optimal control models described in the previous paragraph only consider the maximization of the final 1,3-PD yield. However, in actual operation, it is also important to consider the cost associated with changing the process inputs: large changes to the glycerol and alkali addition rates are difficult (and potentially very costly) to implement in practice. Accordingly, in this paper, we consider a hybrid objective function that takes both 1,3-PD yield and input volatility into account. The optimal control model involves minimizing this hybrid objective function subject to a nonlinear multistage dynamic model for the fed-batch fermentation process, and continuous inequality constraints to reflect operational requirements. Since the governing multistage dynamic system is highly nonlinear, numerical techniques are unavoidable for solving the proposed optimal control model. We develop a novel approach based on the constraint transcription method [22], particle swarm optimization [23, 24, 25] and gradient-based nonlinear programming [26, 27].

The remainder of this paper is organized as follows. In Section 2, we present a nonlinear multistage dynamic model to describe the microbial fed-batch fermentation process. Next, in Section 3, we introduce a novel optimal control model with hybrid objective function consisting of two terms: the first term encourages high 1,3PD yield; the second term penalizes variation in the input feeding rate (the control variable for the process). By using the constraint transcription method, we obtain an approximate parameter optimization problem, which can be solved using the combined particle swarm and gradient-based optimization algorithm described in Section 4. Finally, in Section 5, we present the results from our extensive numerical simulations.

\section{Process dynamics}

We consider the fed-batch fermentation process described in [1] for converting glycerol to 1,3-PD. The process model is derived by ignoring time-delay effects and non-uniform space distribution. For batch mode, the mass balance relationships for biomass, substrate and reaction products can be expressed by the following differential equations:

$$
\begin{aligned}
\dot{x}_{1}(t) & =\mu(t) x_{1}(t), \\
\dot{x}_{2}(t) & =-q_{2}(t) x_{1}(t), \\
\dot{x}_{i}(t) & =q_{i}(t) x_{1}(t), \quad i=3,4,5,
\end{aligned}
$$

where $t$ denotes process time (in hours); and $x_{i}(t)$, $i=1,2,3,4,5$, are, respectively, the concentrations of biomass, glycerol, 1,3-PD, acetic acid and ethanol $\left(x_{1}(t)\right.$ is measured in $\mathrm{g} \mathrm{L}^{-1}$ and the other state variables are measured in mmol L${ }^{-1}$ ). Furthermore, $\mu(t)$ is the specific growth rate of cells $\left(\right.$ in $\left.\mathrm{h}^{-1}\right) ; q_{2}(t)$ is the specific consumption rate of substrate $\left(\right.$ in $\left.^{-1}\right)$; and $q_{i}(t)$, $i=3,4,5$, are, respectively, the specific formation rates of the reaction products 1,3-PD, acetic acid and ethanol.

For feeding mode, the mass balance relationships can be expressed by

$$
\begin{aligned}
& \dot{x}_{1}(t)=(\mu(t)-D(t)) x_{1}(t), \\
& \dot{x}_{2}(t)=D(t)\left(\frac{\rho_{g}}{R+1}-x_{2}(t)\right)-q_{2}(t) x_{1}(t), \\
& \dot{x}_{i}(t)=q_{i}(t) x_{1}(t)-D(t) x_{i}(t), \quad i=3,4,5,
\end{aligned}
$$

where $D(t)$ denotes the dilution rate at time $t, \rho_{g}$ denotes the concentration of glycerol in the input feed, and $R$ is the ratio of alkali to glycerol in the input feed.

Based on the work in [1], the specific growth rate of cells can be expressed as follows:

$$
\mu(t):=\frac{\Delta_{1} x_{2}(t)}{x_{2}(t)+k_{1}} \prod_{l=2}^{5}\left(1-\frac{x_{l}(t)}{x_{l}^{*}}\right)^{n_{l}},
$$

where $\Delta_{1}$ is the maximum specific growth rate; $x_{l}^{*}$, $l=2,3,4,5$, are the maximum residual concentrations of substrate and reaction products; $k_{1}$ is the Monod saturation constant; and $n_{l}, l=2,3,4,5$, are given exponents. Moreover the specific consumption rate of substrate can be expressed as follows:

$$
q_{2}(t):=m_{2}+\frac{\mu(t)}{Y_{2}}+\frac{\Delta_{2} x_{2}(t)}{x_{2}(t)+k_{2}},
$$


where $m_{2}$ is the maintenance term of substrate consumption under substrate-limited conditions; $Y_{2}$ is the maximum growth yield; $\Delta_{2}$ is the maximum increment of substrate consumption rate under substrate-sufficient conditions; and $k_{2}$ is the saturation constant for substrate.

The specific formation rates of 1,3-PD and acetic acid are defined as

$$
q_{i}(t):=m_{i}+Y_{i} \mu(t)+\frac{\Delta_{i} x_{2}(t)}{x_{2}(t)+k_{i}}, \quad i=3,4,
$$

where $m_{3}$ and $m_{4}$ are the maintenance terms of 1,3-PD and acetic acid formations under substrate-limited conditions; $Y_{3}$ and $Y_{4}$ are the maximum 1,3-PD and acetic acid yields; $\Delta_{3}$ and $\Delta_{4}$ are the maximum increments of 1,3-PD and acetic acid formation rates under substratesufficient conditions; and $k_{3}$ and $k_{4}$ are the saturation constants for 1,3-PD and acetic acid.

The specific formation rate of ethanol can be expressed by

$$
q_{5}(t):=q_{2}(t)\left(\frac{c_{1}}{c_{2}+\mu(t) x_{2}(t)}+\frac{c_{3}}{c_{4}+\mu(t) x_{2}(t)}\right),
$$

where $c_{1}, c_{2}, c_{3}$ and $c_{4}$ are given parameters.

Furthermore, the dilution rate $D(t)$ and volume $V(t)$ are given by

$$
\begin{aligned}
& D(t):=\frac{u(t)}{V(t)}, \\
& V(t):=V_{0}+\int_{0}^{t} u(s) d s,
\end{aligned}
$$

where $V_{0}$ denotes the initial volume of solution in the fermentor and $u(t)$ denotes the input feeding rate.

Let

$$
f^{b}(t, x(t)):=\left(\begin{array}{c}
\mu(t) x_{1}(t) \\
-q_{2}(t) x_{1}(t) \\
q_{3}(t) x_{1}(t) \\
q_{4}(t) x_{1}(t) \\
q_{5}(t) x_{1}(t)
\end{array}\right)
$$

and

$$
f^{c}(t, x(t), D(t)):=\left(\begin{array}{c}
(\mu(t)-D(t)) x_{1}(t) \\
D(t)\left(\frac{\rho_{g}}{R+1}-x_{2}(t)\right)-q_{2}(t) x_{1}(t) \\
q_{3}(t) x_{1}(t)-D(t) x_{3}(t) \\
q_{4}(t) x_{1}(t)-D(t) x_{4}(t) \\
q_{5}(t) x_{1}(t)-D(t) x_{5}(t)
\end{array}\right),
$$

where $x(t):=\left(x_{1}(t), x_{2}(t), x_{3}(t), x_{4}(t), x_{5}(t)\right)^{\top}$ is the state vector and $D(t)$ is the dilution rate defined above, which depends on the input feeding rate.
Let $T$ be the terminal time. Furthermore, let $N$ be the number of feeding modes. Since the fed-batch process starts and ends in batch mode, there are exactly $2 N+1$ modes in total ( $N$ feeding modes, $N+1$ batch modes). The complete fed-batch process can be expressed as the following nonlinear multistage dynamic system [1]:

$\dot{x}(t)=\left\{\begin{array}{l}f^{b}(t, x(t)), \quad t \in\left[t_{2 j}, t_{2 j+1}\right), \quad j=0, \ldots, N, \\ f^{c}(t, x(t), D(t)), \quad t \in\left[t_{2 j-1}, t_{2 j}\right), \quad j=1, \ldots, N,\end{array}\right.$ $x(0)=x_{0}$,

where $t_{0}, t_{1}, \ldots, t_{2 N+1}$, are given switching times such that $0=t_{0}<t_{1}<t_{2}<\cdots<t_{2 N+1}=T$; and $x_{0}$ is a given initial state vector. Note that $t_{2 j}$ marks the end of feeding mode and the beginning of batch mode, i.e., the glycerol and alkali feeds are shut off at $t=t_{2 j}$.

Clearly, during batch mode, the feeding rate is zero:

$$
u(t)=0, \quad t \in\left[t_{2 j}, t_{2 j+1}\right), \quad j=0, \ldots, N .
$$

During feeding mode, the feeding rate is subject to the following constraint:

$$
a_{j} \leq u(t) \leq b_{j}, \quad t \in\left[t_{2 j-1}, t_{2 j}\right), \quad j=1, \ldots, N,
$$

where $a_{j}$ and $b_{j}$ are lower and upper bounds for the input feeding rate during the $j$ th feeding mode.

Since the concentrations of biomass, glycerol and reaction products must be restricted to biologically meaningful ranges, we impose the following path constraints:

$$
x_{* i} \leq x_{i}(t) \leq x_{i}^{*}, \quad t \in[0, T], \quad i=1,2,3,4,5,
$$

where $x_{* i}, i=1,2,3,4,5$, are, respectively, the lower concentration thresholds for cell growth for biomass, glycerol, 1,3-PD, acetic acid and ethanol, and $x_{i}^{*}, i=$ $1,2,3,4,5$, are the corresponding upper concentration thresholds (as used in the formula for $\mu(t)$ ).

\section{Optimal control model}

We assume that the input feeding rate is constant during each feeding mode. Then

$$
u(t)=\sigma_{j}, \quad t \in\left[t_{2 j-1}, t_{2 j}\right), \quad j=1, \ldots, N,
$$

where $\sigma_{j}$ denotes the input feeding rate during the $j$ th feeding mode. Hence, during the feeding mode $\left[t_{2 j-1}, t_{2 j}\right)$, the volume $V(t)$ becomes

$$
\begin{aligned}
V(t) & =V_{0}+\sum_{l=1}^{j-1} \int_{t_{2 l-1}}^{t_{2 l}} u(s) d s+\int_{t_{2 j-1}}^{t} u(s) d s \\
& =V_{0}+\sum_{l=1}^{j-1} \sigma_{l}\left(t_{2 l}-t_{2 l-1}\right)+\sigma_{j}\left(t-t_{2 j-1}\right) .
\end{aligned}
$$


Furthermore, the dilution rate during the feeding mode $\left[t_{2 j-1}, t_{2 j}\right)$ becomes

$$
D(t)=\frac{\sigma_{j}}{V(t)} .
$$

The goal of glycerol bioconversion is to maximize the concentration of 1,3-PD at the terminal time. Thus, we consider the following optimization objective:

Objective 1: $\max x_{3}(T)$.

Objective 1 is not the only consideration in practical fermentation processes; the cost of changing the feeding rate signal must also be considered. Indeed, in real fermentation processes, making large changes to the feeding rate may adversely affect process stability.

We use the concept of total variation to measure feeding rate volatility. The total variation of the control variable $u:[0, T] \rightarrow R$ is defined by

$$
\bigvee_{0}^{T} u=\sup \sum_{r=1}^{m}\left|u\left(\eta_{r}\right)-u\left(\eta_{r-1}\right)\right|,
$$

where the supremum is taken over all partitions satisfying

$$
0=\eta_{0}<\eta_{1}<\cdots<\eta_{m-1}<\eta_{m}=T .
$$

Using the results in [28], it can be shown that

$$
\bigvee_{0}^{T} u=2 \sum_{j=1}^{N} \sigma_{j}
$$

To measure changes in the feeding rate signal, we define an additional optimization objective as follows:

$$
\text { Objective 2: } \min \bigvee_{0}^{T} u=2 \sum_{j=1}^{N} \sigma_{j} .
$$

Choosing a weight factor $\alpha \geq 0$ and combining Objectives 1 and 2 leads to the following nonlinear multistage optimal control problem, which we call Problem 1:

$$
\begin{array}{ll}
\min & -x_{3}(T)+2 \alpha \sum_{j=1}^{N} \sigma_{j} \\
\text { s.t. } & a_{j} \leq \sigma_{j} \leq b_{j}, \quad j=1, \ldots, N, \\
& x_{* i} \leq x_{i}(t) \leq x_{i}^{*}, \quad t \in[0, T], \quad i=1, \ldots, 5 .
\end{array}
$$

\section{Numerical solutions}

The main difficulty with solving Problem 1 is the "infinite index" path constraints on the concentration levels. These constraints must hold at every point in the time horizon and thus present a major computational challenge. To address this challenge, we will apply the constraint transcription method as described in [1, 22].

\subsection{Constraint transcription}

First, note that the concentration bounds can be written in equivalent form as

$$
\begin{aligned}
& \sum_{i=1}^{5} \int_{0}^{T} \min \left\{0, x_{i}^{*}-x_{i}(t)\right\} d t \\
& +\sum_{i=1}^{5} \int_{0}^{T} \min \left\{0, x_{i}(t)-x_{* i}\right\} d t=0 .
\end{aligned}
$$

However, since the $\min \{0, \cdot\}$ function is nondifferentiable, constraint (1) is not suitable for gradientbased optimization techniques (which we will exploit later to design a solution algorithm). We therefore consider the following smooth approximation of the $\min \{0, \cdot\}$ function:

$$
\min \{0, \eta\} \approx \varphi_{\varepsilon}(\eta)
$$

where

$$
\varphi_{\varepsilon}(\eta):= \begin{cases}\eta, & \text { if } \eta<-\varepsilon \\ -(\eta-\varepsilon)^{2} / 4 \varepsilon, & \text { if }-\varepsilon \leq \eta \leq \varepsilon \\ 0, & \text { otherwise }\end{cases}
$$

and $\varepsilon>0$ is an adjustable parameter. It is easy to verify that $\varphi_{\varepsilon}$ is continuously differentiable and non-positive. Using $\varphi_{\varepsilon}$, constraint (1) is approximated by

$$
\sum_{i=1}^{5} \int_{0}^{T}\left\{\varphi_{\varepsilon}\left(x_{i}^{*}-x_{i}(t)\right)+\varphi_{\varepsilon}\left(x_{i}(t)-x_{* i}\right)\right\} d t \geq-\gamma
$$

where $\gamma>0$ is an adjustable parameter. Hence, with this approximation scheme, the concentration bounds in Problem 1 are approximated by the single canonical constraint (2). This constraint is a standard constraint and can be readily handled using the computational algorithm described later in Section 4.2.

Problem 1 can now be approximated by the following optimization problem called Problem 2:

$$
\begin{array}{ll}
\min & -x_{3}(T)+2 \alpha \sum_{j=1}^{N} \sigma_{j} \\
\text { s.t. } & \sum_{i=1}^{5} \int_{0}^{T}\left\{\varphi_{\varepsilon}\left(x_{i}^{*}-x_{i}(t)\right)+\varphi_{\varepsilon}\left(x_{i}(t)-x_{* i}\right)\right\} d t \geq-\gamma, \\
& a_{j} \leq \sigma_{j} \leq b_{j}, \quad j=1, \ldots, N .
\end{array}
$$




\subsection{Algorithm description}

Problem 2 is a smooth optimal parameter selection problem that can be solved using the gradient-based optimization methods in [27]. These methods, however, are only designed to find local optimal solutions. Hence, in this section, we combine gradient-based optimization with particle swarm optimization (PSO) to develop a global search algorithm for solving Problem 2.

PSO was originally developed by Kennedy and Eberhart as an evolutionary computational technique based on smarm intelligence [23]. In recent years, PSO has received increasing attention in the optimization field [24, 25]. Normally, the traditional PSO method is designed to deal with unconstrained optimization problems. Problem 2, however, involves a variety of different constraints, including a nonlinear inequality constraint on the state variables.

Throughout this section, we let $\sigma \in \mathbb{R}^{N}$ denote the collection of control values $\sigma_{j}, j=1, \ldots, N$, i.e., $\sigma$ is the decision vector in Problem 2. The input parameters for our algorithm are defined below:

- $M$ is the total number of particles in the swarm.

- $\epsilon$ is a tolerance parameter.

- $L_{\min }$ and $L_{\max }$ are lower and upper bounds for the number of iterations.

- $L_{\epsilon}$ is an integer for testing convergence (if the optimal objective value does not improve sufficiently over $L_{\epsilon}$ iterations, then we switch from particle swarm to gradient-based search).

- $\omega_{\min }$ and $\omega_{\max }$ are the minimum and maximum inertia weights.

- $\pi_{1}$ and $\pi_{2}$ are the acceleration coefficients.

The algorithm also maintains the following variables as the iterations proceed:

- $l$ is the iteration index.

- $\sigma^{m, *}$ is the best control strategy found by the $m$ th individual particle.

- $\sigma^{*}$ is the best control strategy found by the swarm.

- $J^{m, *}$ is the best objective value found by the $m$ th individual particle.

- $J_{l}^{*}$ is the best objective value found by the swarm over the first $l$ iterations.
We use the following notation to represent the objective function and the left-hand side of the first constraint in Problem 2:

$$
\begin{aligned}
G(\sigma) & :=-x_{3}(T)+2 \alpha \sum_{j=1}^{N} \sigma_{j} \\
H(\sigma) & :=\sum_{i=1}^{5} \int_{0}^{T}\left\{\varphi_{\varepsilon}\left(x_{i}^{*}-x_{i}(t)\right)+\varphi_{\varepsilon}\left(x_{i}(t)-x_{* i}\right)\right\} d t .
\end{aligned}
$$

Our algorithm exploits the gradients of $G(\cdot)$ and $H(\cdot)$ with respect to $\sigma$. These gradients can be computed using the methods in [27].

Algorithm 1. Solves Problem 2 using gradient-based optimization and PSO techniques.

1. Set $1 \rightarrow l,+\infty \rightarrow J^{m, *}$ and $+\infty \rightarrow J_{0}^{*}$.

2. Form a sequence $\left\{\sigma^{m}\right\}_{m=1}^{M}$ of admissible particles by randomly sampling over the rectangular region defined by the box constraints

$$
a_{j} \leq \sigma_{j} \leq b_{j}, \quad j=1, \ldots, N
$$

3. Form a sequence $\left\{v^{m}\right\}_{m=1}^{M}$ of particle velocities by randomly sampling over a suitable rectangular region.

4. For each $m=1, \ldots, M$, check the value of $H\left(\sigma^{m}\right)$. If $H\left(\sigma^{m}\right)<-\gamma\left(\right.$ i.e., $\sigma^{m}$ is infeasible for Problem 2), then perform a gradient-ascent search (maximizing $H(\cdot)$ ) to obtain a feasible point $\tilde{\sigma}^{m}$ for Problem 2 and set $\tilde{\sigma}^{m} \rightarrow \sigma^{m}$.

5. For each $m=1, \ldots, M$, update the optimal strategy for the mth particle: if $G\left(\sigma^{m}\right)<J^{m, *}$, then set $G\left(\sigma^{m}\right) \rightarrow J^{m, *}$ and $\sigma^{m} \rightarrow \sigma^{m, *}$.

6. Update the global optimal strategy: if $\min G\left(\sigma^{m}\right)<$ $J_{l-1}^{*}$, then set $\min G\left(\sigma^{m}\right) \rightarrow J_{l}^{*}$ and $\arg \min G\left(\sigma^{m}\right) \rightarrow$ $\sigma^{*}$; otherwise, set $J_{l-1}^{*} \rightarrow J_{l}^{*}$.

7. If $l=L_{\max }$, or if $l \geq L_{\min }$ and $\left|J_{l}^{*}-J_{l-L_{\epsilon}}^{*}\right|<\epsilon$, then use gradient-based optimization methods (initialized with $\sigma^{*}$ as the starting point) to determine an optimal solution and stop. Otherwise, go to Step 8.

8. Calculate the inertia weight for the particles:

$$
\omega_{\max }-\frac{\omega_{\max }-\omega_{\min }}{L_{\max }-2}(l-1) \rightarrow \omega .
$$




\begin{tabular}{ccccccccc}
\hline & & & \multicolumn{2}{c}{ Number of modes } & & \multicolumn{3}{c}{ Mode duration (s) } \\
\cline { 7 - 8 } Phase & Start time $(\mathrm{h})$ & End time $(\mathrm{h})$ & Feeding & Batch & & Feeding & Batch & Final Batch \\
\hline 1 & 5.33 & 6.13 & 29 & 29 & & 5 & 95 & 75 \\
2 & 6.13 & 7.15 & 37 & 37 & & 7 & 93 & 65 \\
3 & 7.15 & 7.83 & 25 & 25 & & 8 & 92 & 40 \\
4 & 7.83 & 8.83 & 36 & 36 & & 8 & 92 & 92 \\
5 & 8.83 & 12.16 & 120 & 120 & & 7 & 93 & 81 \\
6 & 12.16 & 15.83 & 133 & 133 & & 6 & 94 & 6 \\
7 & 15.83 & 18.10 & 82 & 82 & & 4 & 96 & 68 \\
8 & 18.10 & 19.83 & 63 & 63 & & 3 & 97 & 25 \\
9 & 19.83 & 23.83 & 144 & 144 & & 2 & 98 & 98 \\
10 & 23.83 & 24.16 & 12 & 12 & & 1 & 99 & 87 \\
\hline
\end{tabular}

Table 1: Phase characteristics for the test scenario in Section 5. Each phase consists of a series of identical 100 second feeding-batch combinations plus a final feeding-batch combination that may have a different duration. Within each phase, all feeding modes have the same duration and all batch modes except the last have the same duration.

9. For each $m=1, \ldots, M$, update the position and velocity of the mth particle:

$$
\begin{aligned}
& \omega v^{m}+\pi_{1} r_{1}\left(\sigma^{m, *}-\sigma^{m}\right)+\pi_{2} r_{2}\left(\sigma^{*}-\sigma^{m}\right) \rightarrow v^{m}, \\
& \sigma^{m}+v^{m} \rightarrow \sigma^{m},
\end{aligned}
$$

where $r_{1}$ and $r_{2}$ are random numbers in $[0,1]$.

10. For each $m=1, \ldots, M$, check whether $\sigma^{m}$ violates the box constraints in Step 2: For any component of $\sigma^{m}$ that violates its lower bound, update that component to be equal to the lower bound; for any component that violates its upper bound, update that component to be equal to the upper bound.

\section{Set $l+1 \rightarrow l$ and return to Step 4 .}

\section{Numerical results and discussion}

To test Algorithm 1, we considered the test scenario in [1], which is based on real experimental data. In this test scenario, the fed-batch process consists of an initial batch mode followed by 10 phases, where each phase involves an equal number of feeding and batch modes operating in succession. Within each phase, all feeding modes have the same duration, and all batch modes except the last have duration 100 seconds minus the feeding mode duration. The characteristics of each phase are given in Table 1. The entire fed-batch process operates for $T=24.16$ hours, with the initial batch mode operating for 5.33 hours. For the initial state values, the initial concentration of biomass is $0.1115 \mathrm{~g} \mathrm{~L}^{-1}$, the initial concentration of glycerol is
$495 \mathrm{mmol} \mathrm{L}^{-1}$, and the initial concentrations of 1,3$\mathrm{PD}$, acetic acid and ethanol are all $0 \mathrm{mmol} \mathrm{L}^{-1}$. Hence, $x_{0}=(0.1115,495,0,0,0)^{\top}$. Furthermore, the initial volume in the fermentor is $V_{0}=5 \mathrm{~L}$, the concentration of glycerol in the input feed is $\rho_{g}=10762 \mathrm{mmol}$ $\mathrm{L}^{-1}$, and the ratio of alkali to glycerol in the input feed is $R=0.75$.

\subsection{Parameter identification}

Before solving the optimal control problem, the values of the model constants in the dynamic model must be determined. To do this, we solved the following least-squares optimization problem corresponding to the experimental data in [29]:

$$
\min \sum_{i=1}^{5} \sum_{r=\kappa_{i}}^{p} \varsigma_{i}\left(x_{i}\left(\tau_{r}\right)-\hat{x}_{i}^{r}\right)^{2},
$$

where $x_{i}\left(\tau_{r}\right)$ is the predicted value of state $i$ at sample time $\tau_{r}, \hat{x}_{i}^{r}$ is the measured value of state $i$ at sample time $\tau_{r}$, and the constants $\varsigma_{1}, \varsigma_{2}, \varsigma_{3}, \varsigma_{4}$ and $\varsigma_{5}$ are weight factors. The aim here is to choose the tunable model parameters $m_{i}, i=2,3,4 ; Y_{i}, i=2,3,4 ; \Delta_{i}, i=2,3,4$; $k_{i}, i=1,2,3,4$; and $c_{i}, i=1,2,3,4$ to minimize (3), which measures the discrepancy between the model and the experimental data. Note that $\Delta_{1}, n_{2}, n_{3}, n_{4}, n_{5}, x_{1}^{*}$, $x_{2}^{*}, x_{3}^{*}, x_{4}^{*}$, and $x_{5}^{*}$ have well-defined values and do not need to be tuned. As in [29], we use $\Delta_{1}=0.67, n_{2}=1$, $n_{3}=n_{4}=n_{5}=3, x_{1}^{*}=6, x_{2}^{*}=2039, x_{3}^{*}=1036$, $x_{4}^{*}=1026$, and $x_{5}^{*}=361$.

The test data in [29] contains 12 data points $(p=12)$ as shown in Table 2. This data was generated using a constant feeding rate in each phase, the values of which 


\begin{tabular}{ccccccc}
\hline$r$ & $\tau_{r}$ & $\hat{x}_{1}^{r}\left(\mathrm{~g} \mathrm{~L}^{-1}\right)$ & $\hat{x}_{2}^{r}\left(\mathrm{mmol} \mathrm{L}^{-1}\right)$ & $\hat{x}_{3}^{r}\left(\mathrm{mmol} \mathrm{L}^{-1}\right)$ & $\hat{x}_{4}^{r}\left(\mathrm{mmol} \mathrm{L}^{-1}\right)$ & $\hat{x}_{5}^{r}\left(\mathrm{mmol} \mathrm{L}^{-1}\right)$ \\
\hline 1 & 2.00 & 0.50 & 434.35 & - & - & - \\
2 & 4.00 & 1.31 & 323.26 & 69.74 & 102.50 & - \\
3 & 5.83 & 2.60 & 182.61 & 168.82 & 164.83 & - \\
4 & 7.83 & 3.62 & 202.07 & 301.84 & 198.67 & - \\
5 & 9.83 & 4.90 & 212.17 & 411.58 & 282.50 & - \\
6 & 11.83 & 4.85 & 219.24 & 488.82 & 213.50 & 43.26 \\
7 & 13.83 & 5.42 & 209.13 & 673.16 & 176.67 & 53.04 \\
8 & 15.83 & 5.23 & 247.50 & 705.79 & 196.33 & 90.43 \\
9 & 17.83 & 4.92 & 242.39 & 745.26 & 187.50 & 103.91 \\
10 & 19.83 & 5.19 & 252.50 & 862.27 & 238.33 & 151.74 \\
11 & 21.83 & 5.07 & 242.39 & 824.47 & 204.83 & 101.30 \\
12 & 23.83 & 4.91 & 222.17 & 797.24 & 234.00 & 147.61 \\
\hline
\end{tabular}

Table 2: Experimental data used in Section 5.1.

\begin{tabular}{ccccccc}
\hline & Phases 1-2 & Phases 3-4 & Phases 5-6 & Phase 7 & Phase 8 & Phases 9-10 \\
\hline$u(t)$ & 1.32489 & 1.25489 & 1.32789 & 1.39489 & 1.53487 & 1.60487 \\
\hline
\end{tabular}

Table 3: Feeding rates (in $\mathrm{L} \mathrm{h}^{-1}$ ) for the experimental data used in Section 5.1.

are given in Table 3. For the least-squares error function (3), the weight factors $\varsigma_{i}, i=1,2,3,4,5$, were chosen as follows:

$$
\begin{aligned}
& \varsigma_{1}=1.0 \times 10^{2}, \quad \varsigma_{2}=1.5 \times 10^{-2}, \quad \varsigma_{3}=1.5 \times 10^{-2}, \\
& \varsigma_{4}=1.0 \times 10^{-2}, \quad \varsigma_{5}=1.0 \times 10^{-2} .
\end{aligned}
$$

We solved the estimation problem using the Fortran optimization solver NLPQLP [30] combined with the gradient computation procedure in [31]. The optimal parameters obtained are given in Table 4 . The corresponding state trajectories are shown in Figure 1.

\subsection{Optimal control strategy}

Based on the dynamic model with optimized parameters from Section 5.1, the next step is to determine the optimal control strategy. For the optimal control problem, the lower concentration thresholds for biomass, glycerol, 1,3-PD, acetic acid, and ethanol are given as follows:

$$
x_{* 1}=0.01, x_{* 2}=15, x_{* 3}=0, x_{* 4}=0, x_{* 5}=0 .
$$

The upper concentration thresholds are as defined in Section 5.1. Recall that these thresholds define the path constraints. The lower and upper bounds for the feeding rate are listed in Table 5.
Our implementation of Algorithm 1 uses the Fortran software NLPQLP [30] to perform the gradient-based optimization process in Step 7. For the parameters in Algorithm 1, we used the following values:

$$
\begin{gathered}
M=100, \epsilon=0.01, L_{\min }=1, L_{\max }=1000, \\
L_{\epsilon}=5, \pi_{1}=\pi_{2}=1, \omega_{\min }=1, \omega_{\max }=100 .
\end{gathered}
$$

\begin{tabular}{ccc}
\hline Phases & $a_{j}$ & $b_{j}$ \\
\hline $1-2$ & 0.6624 & 1.9873 \\
$3-4$ & 0.6274 & 1.8823 \\
$5-6$ & 0.6624 & 1.9873 \\
7 & 0.6974 & 2.0923 \\
8 & 0.7674 & 2.3023 \\
$9-10$ & 0.8024 & 2.4073 \\
\hline
\end{tabular}

Table 5: Lower and upper bounds for the feeding rate in Section 5.2. 


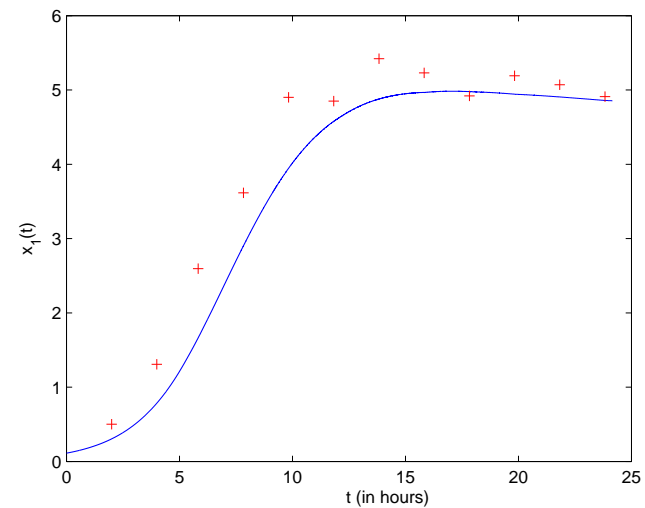

(a) Concentration of biomass (in $\mathrm{g} \mathrm{L}^{-1}$ )

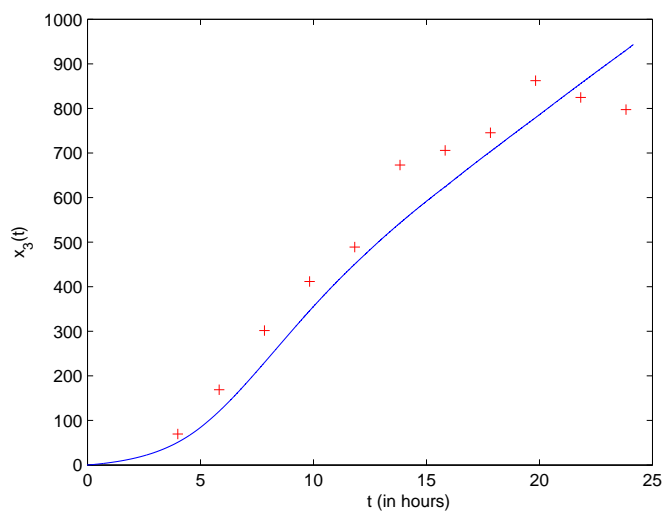

(c) Concentration of 1,3-PD (in $\mathrm{mmol} \mathrm{L}^{-1}$ )

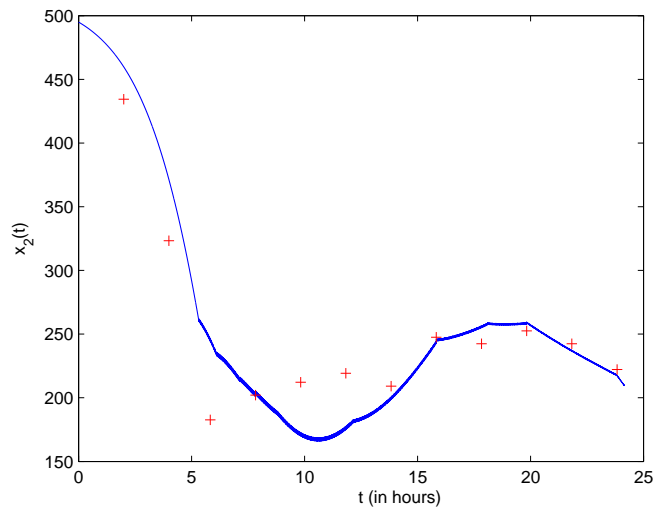

(b) Concentration of glycerol (in $\mathrm{mmol} \mathrm{L}^{-1}$ )

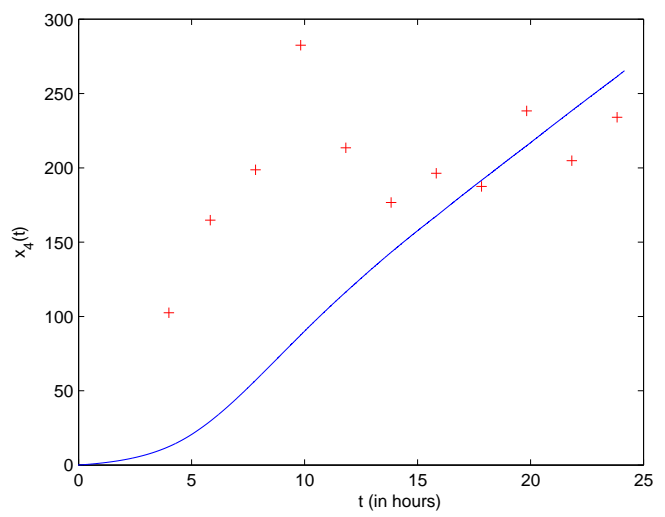

(d) Concentration of acetic acid (in $\mathrm{mmol} \mathrm{L}^{-1}$ )

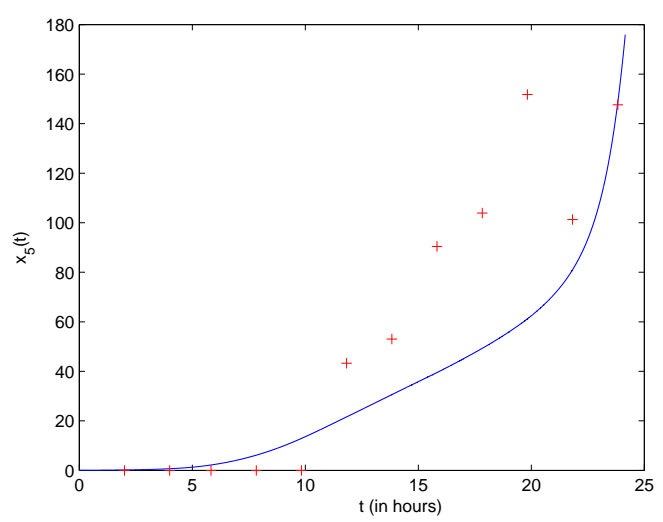

(e) Concentration of ethanol (in $\mathrm{mmol} \mathrm{L}^{-1}$ )

Figure 1: Optimal trajectories for the parameter tuning problem in Section 5.1. The blue lines represent the model predictions corresponding to the optimal parameter estimates; the red crosses represent the experimental data. 


\begin{tabular}{cccccc}
\hline$i$ & $m_{i}$ & $Y_{i}$ & $\Delta_{i}$ & $k_{i}$ & $c_{i}$ \\
\hline 1 & - & - & 0.6700 & 0.056 & 0.045 \\
2 & 0.3420 & 0.0060 & 8.8703 & 30.0420 & 0.01475 \\
3 & -0.6120 & 57.9232 & 9.2967 & 3.1000 & 1.1254 \\
4 & -0.1940 & 12.9718 & 2.8439 & 3.1420 & 10.0900 \\
\hline
\end{tabular}

Table 4: Optimal parameter estimates for Section 5.1.

\begin{tabular}{ccc}
\hline & & ${ }^{T}$ \\
$\alpha$ & $x_{3}(T)$ & $\bigvee_{0} u$ \\
\hline 0 & 971.32 & 1353.68 \\
0.1 & 966.99 & 1267.27 \\
0.2 & 947.81 & 1136.21 \\
0.3 & 939.57 & 1102.75 \\
0.4 & 931.13 & 1078.62 \\
0.5 & 924.82 & 1064.30 \\
0.6 & 922.73 & 1060.43 \\
0.7 & 922.04 & 1059.37 \\
0.8 & 921.42 & 1058.92 \\
0.9 & 921.28 & 1058.58 \\
1 & 921.28 & 1058.43 \\
\hline
\end{tabular}

Table 6: Numerical results for Section 5.2.

Using Algorithm 1 (implemented within Fortran), we solved Problem 2 for different values of $\alpha$. The optimal 1,3-PD yields and feeding rate variations are given in Table 6. The results show that when $\alpha$ is increased, the control variation can be reduced with little change to 1,3-PD yield. For example, the difference in 1,3-PD yield between $\alpha=0$ and $\alpha=1$ is around $5.15 \%$, but the difference in control variation is around $21.81 \%$. The optimal state trajectories for $\alpha=0$ and $\alpha=1$ are shown in Figure 2.

\section{Conclusion}

This paper has studied a nonlinear multistage dynamic system for describing the fed-batch fermentation process used in 1,3-PD production. To maximize the productivity of the process, we formulated a novel optimization model in which the objective function measures both the final yield of 1,3-PD and the cost of changing the substrate feed. Large changes in the substrate feed may cause potential hazards, or could even be impossible to implement in practice. Our hybrid cost function is designed to ensure that an appropriate balance is struck between 1,3-PD yield and implementa- tion cost. To solve the proposed non-standard optimization model, a computational method based on constraint transcription, particle swarm optimization and gradientbased nonlinear programming was developed. The numerical results in Section 5 show that the method is successful at producing high-quality control strategies with low feeding rate volatility.

\section{Acknowledgements}

This work was supported by the National Natural Science Foundation of China (Grants 11171050, 11371164), the National Natural Science Foundation for the Youth of China (Grants 11301081, 11401073, 11201267), and the Fundamental Research Funds for Central Universities in China (Grant DUT15LK25).

[1] C.Y. Liu, Z.H. Gong, E.M. Feng, H.C. Yin, Modelling and optimal control for nonlinear multistage dynamical system of microbial fed-batch culture, J. Ind. Manag. Optim. 5 (2009) 835-850.

[2] K. Menzel, A.P. Zeng, W.D. Deckwer, High concentration and productivity of 1,3-propanediol from continuous fermentation of glycerol by Klebsiella pneumoniae, Enzym. Microb. Technol. 20 (1997) 82-86.

[3] M. Hartlep, W. Hussmann, N. Prayitno, I. Meynial-Salles, A.P. Zeng, Study of two-stage processes for the microbial production of 1,3-propanediol from glucose, Appl. Microbiol. Biotechnol. 60 (2002) 60-66.

[4] H. Biebl, A.P. Zeng, K. Menzel, W.D. Deckwer, Fermentation of glycerol to 1,3-propanediol and 2,3-butanediol by Klebsiella pneumoiae, Appl. Microbiol. Biotechnol. 50 (1998) 24-29.

[5] A. Reimann, H. Biebl, W.D. Deckwer, Production of 1,3propanediol by Clostridium butyrium in continuous culture with cell recycling, Appl. Microbiol. Biotechnol. 49 (1998) 359-363.

[6] A.P. Zeng, H. Biebl, Bulk chemicals from biotechnology: the case of 1,3-propanediol production and the new trends, Adv. Biochem. Eng. Biotechnol. 74 (2002) 239-259.

[7] Z.L. Xiu, A.P. Zeng, L.J. An, Mathematical modelling of kinetics and research on multiplicity of glycerol bioconversion to 1,3-propanediol, J. Dalian Univ. Technol. 4 (2000) 428-433.

[8] J.X. Ye, H.L. Xu, E.M. Feng, Z.L. Xiu, Optimization of a fedbatch bioreactor for 1,3-propanediol production using hybrid nonlinear optimal control, J. Process Control 24 (2014) 15561569.

[9] A.P. Zeng, Pathway and kinetic analysis of 1,3-propanediol production from glycerol fermentation by Clostridium butyricum, Bioprocess Eng. 14 (1996) 169-175.

[10] A.P. Zeng, A. Rose, H. Biebl, C. Tag, B. Guenzel, W.D. Deckwer, Multiple product inhibition and growth modeling of 


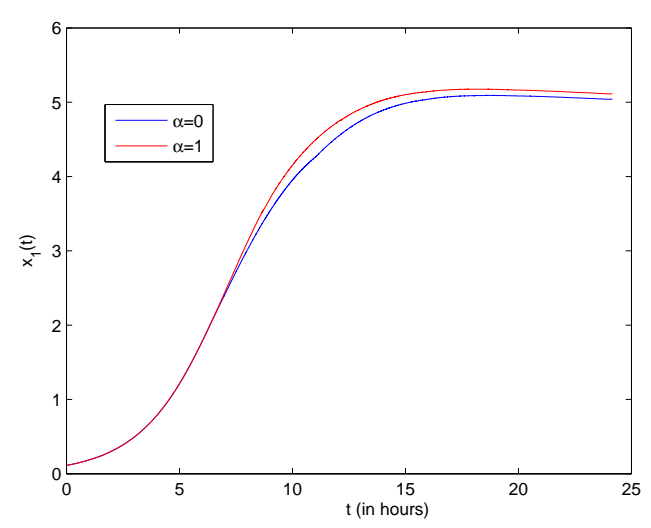

(a) Concentration of biomass (in $\mathrm{g} \mathrm{L}^{-1}$ )

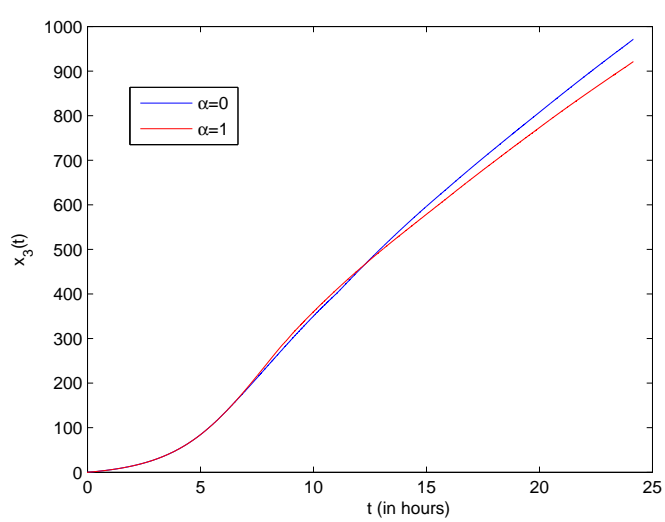

(c) Concentration of 1,3-PD (in $\mathrm{mmol} \mathrm{L}^{-1}$ )

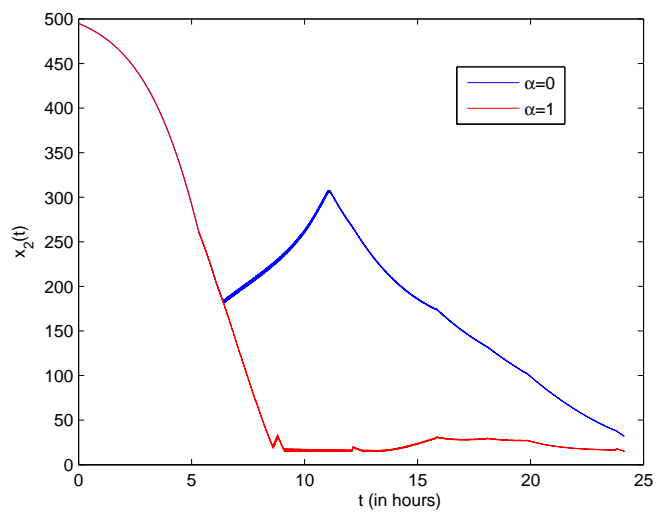

(b) Concentration of glycerol (in $\mathrm{mmol} \mathrm{L}^{-1}$ )

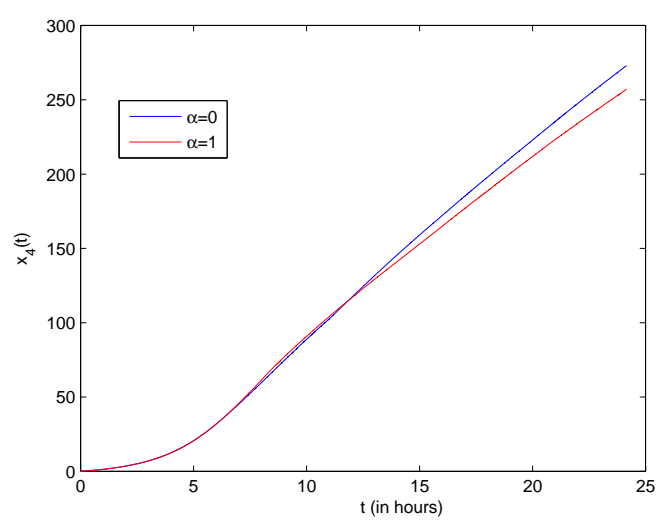

(d) Concentration of acetic acid (in $\mathrm{mmol} \mathrm{L}^{-1}$ )

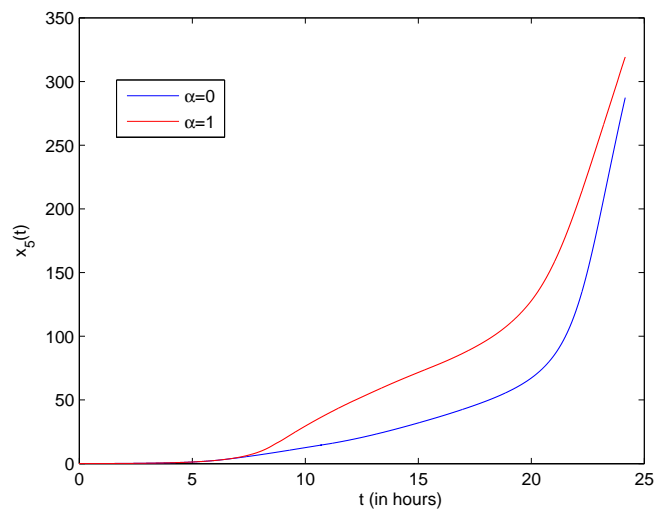

(e) Concentration of ethanol (in $\mathrm{mmol} \mathrm{L}^{-1}$ )

Figure 2: The optimal state trajectories for $\alpha=0$ and $\alpha=1$. 
Clostridium butyricum and Klebsiella pneumoniae in glycerol fermentation, Biotechnol. Bioeng. 44 (1994) 902-911.

[11] K.K. Cheng, J.A. Zhang, D.H. Liu, Y. Sun, H.J. Liu, M.D. Yang, J.M. Xu, Pilot-scale production of 1,3-propanediol using Klebsiella pneumoniae, Process Biochem. 42 (2007) 740-744.

[12] C.X. Gao, E.M. Feng, Z.T. Wang, Z.L. Xiu, Nonlinear dynamical systems of biodissimilation of glycerol to 1,3-propanediol and their optimal controls, J. Ind. Manag. Optim. 1 (2005) $377-$ 388.

[13] C.X. Gao, K.Z. Li, E.M. Feng, Z.L. Xiu, Nonlinear impulsive system of fed-batch culture in fermentative production and its properties, Chaos Solitons Fractals 28 (2006) 271-277.

[14] C.X. Gao, Z.T. Wang, E.M. Feng, Z.L. Xiu, Parameter identification and optimization of process for bio-dissimilation of glycerol to 1,3-propanediol in batch culture, J. Dalian Univ. Technol. 46 (2006) 771-774.

[15] G. Wang, E.M. Feng, Z.L. Xiu, Modelling and parameter identification of microbial bioconversion in fed-batch cultures, J. Process Control 18 (2008) 458-464.

[16] H.Y. Wang, E.M. Feng, Z.L. Xiu, Optimality condition of the nonlinear impulsive system in fed-batch fermentation, Nonlinear Anal. 68 (2008) 12-23.

[17] J.G. Gao, B.Y. Shen, E.M. Feng, Z.L. Xiu, Modelling and optimal control for an impulsive dynamical system in microbial fed-batch culture, Comput. Appl. Math. 32 (2013) 275-290.

[18] C.Y. Liu, Z.H. Gong, Sensitivity analysis and parameter identification for a nonlinear time-delay system in microbial fed-batch process, Appl. Math. Model. 38 (2014) 1449-1463.

[19] Z.H. Gong, C.Y. Liu, E.M. Feng, L. Wang, Y.S. Yu, Modelling and optimization for a switched system in microbial fed-batch culture, Appl. Math. Model. 35 (2011) 3276-3284.

[20] C.Y. Liu, Optimal control of a switched autonomous system with time delay arising in fed-batch processes, IMA J. Appl. Math. 80 (2015) 569-584.
[21] C.Y. Liu, Optimal control for nonlinear dynamical system of microbial fed-batch culture, J. Comput. Appl. Math. 232 (2009) 252-261.

[22] K.L. Teo, L.S. Jennings, Nonlinear optimal control problems with continuous state inequality constraints, J. Optim. Theory Appl. 63 (1989) 1-22.

[23] J. Kennedy, R.C. Eberhart, Particle swarm optimization, in "Proceedings of the 1995 IEEE International Conference on Neural Networks", Perth, Australia, 1995, 1942-1948.

[24] H.Q. Li, L. Li, T.H. Kim, S.L. Xie, An improved PSO-based of harmony search for complicated optimization problems, Int. J. Hybrid Inf. Technol. 1 (2008) 57-64.

[25] J.B. Yu, L.F. Xi, S.J. Wang, An improved particle swarm optimization for evolving feedforward artificial neural networks, Neural Process. Lett. 26 (2007) 217-231.

[26] K.L. Teo, C.J. Goh, K.H. Wong, A Unified Computational Approach to Optimal Control Problems, Longman Scientific and Technical, Essex, 1991.

[27] Q. Lin, R. Loxton, K.L. Teo, The control parameterization method for nonlinear optimal control: a survey, J. Ind. Manag. Optim. 10 (2014) 275-309.

[28] R. Loxton, Q. Lin, K.L. Teo, Minimizing control variation in nonlinear optimal control, Automatica 49 (2013) 2652-2664.

[29] X. Chen, D.J. Zhang, W.T. Qi, S.J. Gao, Z.L. Xiu, P. Xu, Microbial fed-batch production of 1,3-propanediol by Klebsiella pneumoniae under microaerobic conditions, Appl. Microbiol. Biotechnol. 63 (2003) 143-146.

[30] K. Schittkowski, NLPQLP: a Fortran implementation of a sequential quadratic programming algorithm with distributed and non-monotone line search, University of Bayreuth, Bayreuth, 2007.

[31] Q.Q. Chai, R. Loxton, K.L. Teo, C. Yang, A unified parameter identification method for nonlinear time-delay systems, J. Ind. Manag. Optim. 9 (2013) 471-486. 